\title{
Dieter Sterzel
}

\section{Jahre Grundgesetz - Zur verfassungs- rechtlichen Entwicklung der Bundesrepublik seit 1949*}

-Menschenwerk ist immer voller Mängel Kurt Georg Kiesinger ${ }^{1}$

$I$.

In einer Ansprache zum zwanzigjährigen Bestehen des Grundgesetzes hat Bundeskanzler Kiesinger der Bevölkerung über Rundfunk und Fernsehen verkündet, daß "bei uns eine lebendige demokratische, gesellschafliche und politische Ordnung herangewachsen ist, die jedem ein Leben in Freiheit und unserem Volk Wohlstand und soziale Gerechtigkeit gesichert hat $\aleph^{2}$. Bundesinnenminister Benda hat aus dem gleichen Anlaß an jeden, der »unvoreingenommen urteilt«, appelliert, szuzugeben, daß wir die freiheitlichste, die rechtsstaatlichste und die lebendigste demokratische Verfassung haben, die unser Land je besaß « ${ }^{3}$. Nimmt man demgegenüber das Inkraftereten des Grundgesetzes vor 20 Jahren zum Anlaß, sich rüdkblickend der politischen Substanz der verfassungsgestaltenden Grundentscheidungen von 1949 sowie der damit verbundenen Erwartungen zu erinnern und die wichtigsten Stationen der verfassungspolitischen Entwidklung zu bestimmen, so ist festzustellen, daß reziprok zur zunehmenden politischen und ökonomischen Stabilisierung des westdeutschen Ferrschaftssystems der Abbau der im Grundgesetz angelegten freiheitlichen, rechtsstaatlichen und demokratischen Verfassungsprinzipien sich vollzogen hat $t^{32}$. Das Gerede, die im Bundestag vertretenen Parteien »bejahten das Grundgesetz uneingescbränkt (Benda), demaskiert sich auf dem Fintergrund von nunmehr insgesamt 25 Grundgesetzänderungen eindeutig als propagandistisches Ablenkungsmanöver'.

\section{II.}

Die zentralen Entscheidungen des Parlamentarischen Rates sind in den für unabänderlich erklärten Grundsätzen der Art. I und 20 GG fixiert. Hiernach ist leitendes Prinzip der Verfassung die Unantastbarkeit der menschlichen Würde, die zu achten und zu schützen die Verpflichtung und Aufgabe aller staatlichen Gewalt ist. Die Menschenwürde und als deren Konkretisierung die nachfolgenden Grundrechte stehen in engem, verpflichtendem Zusammenhang mit dem Be-

* Uberarbeitete Fassung eines am 20. 5. 2969 in Köln vor dem Republikanischen Club gehaltenen Vortrags.

1 K. G. Kiesinger, 20 Jahre Grundgesetz - 20 Jahre Bundesrepublik Deutschland, Bulletin des Presse- und Informationsamtes der Bundesregierung Nr. 68 vom 28. Mai 1969, S. $58 \mathrm{r}-582$ (S. $58 \mathrm{r}$ ).

2 Bulletin des Presse- und Informationsamtes der Bundesregierung Nr. 68, S. $58 \mathrm{r}$.

3 E. Benda, 20 Jahre Grundgesetz, Bulletin des Presse- und Informationsamtes der Bundesregierung Nr. 67 yom 23. 5. 1969, S. 569-575 (S. 572).

32 Der konservativen Staatsrechtslehre reidien die Verfassungsänderungen allerdings $>$ nicht in die Tiefe, sie haben mehr äußerliche Anpassungen gebracht*; vgl. W. Weber, Die Bundesrepublik und ihre Verfassung an der Schwelle des dritten Jahrzehnts. In DVBI 1969, S. 413-4r9 (S. 4r4).

4 wie Anm. 3, S. 569, Hervorhebung rom Verf. 
kenntnis zu den politischen Strukturprinzipien der rechtsstaatlichen und sozialen Demokratie.

Unbestreitbar war die Abgrenzung gegenüber dem Nationalsozialismus der gemeinsame Nenner, der die demokrarischen Kräfte 1949 miteinander verband ${ }^{3}$. Diese Basis reichte freilich nicht aus, um die politischen $K$ räfte jener Zeit - die Parteien, die Gewerkschaften, die Interessenvertretungen der Unternehmer, die Kirchen und nicht zuletzt die westlichen Besatzungsmächte - auf eine gemeinsame Linie über zentrale machtpolitische Fragen zu verpflichten ${ }^{8}$. Die Entscheidung für die sozialstaatliche Demokratie blieb nicht mehr als ein formelhafter Kompromiß.

Man kann jedoch davon ausgehen, daß das Votum für die parlamentarische Demokratie, sieht man von der Haltung der Kommunistischen Partei einmal ab, einhellig erfolgt ist. Daran ändert nichts die Tatsache, daß diese Entscheidung, ähnlich wie die für den Bundesstaat, von den Besatzungsmächten vorherbestimmt gewesen ist ${ }^{i}$. Es war nur folgerichtig, daß die politischen Parteien, die im Parlamentarischen Rat eine Schlüsselstellung einnahmen, ihre im Zuge des staatlichen Aufbaus nach 1945 mit Unterstützung der Besatzungsmächte gewonnenen Positionen $^{8}$ sicherzustellen suchten. Durch die in Art. 2I GG vorgenommene Institutionalisierung als Organe der politischen Willensbildung ließ sich ihr Herrschaftsanspruch im Rahmen des parlamentarischen Regierungssystems am ehesten realisieren. Die mit dieser Verfassungsnorm verbundene Erwartung hat sich für die beiden großen Parteien, deren quasi-staatliche Monopolstellung u. a. auf die I953 eingeführte $5 \%$ Sperrklausel mit zurüdkzuführen ist, voll erfüllt ${ }^{\theta}$.

5 In einer am 25. Jahrestag des Atrentats auf Hitler in verschiedenen Tages- und Wochenzeitungen (u. a. Frankfurter Rundschau, Stern, Weit der Arbeit, Rheinischer Merkur, Spiegel) veröffentlichten Erklärung der Aktionsgemeinschaft 20. Juli 1944*, der Hermann J. Abs, Th. W. Adorno und roo weitere Persönlichkeiten des öffentlichen Lebens angehören, wird an die Opposition gegen den Nationalsozialismus als der für wnser geistiges und politisches Dasein heute gültigen Legitimacion erinnert. Deutlicher als in diesem Dokument läßr sich die AlibiFunktion der Deklamationen zum 20. Juli nicht belegen. Erschredsend ist freilich das AusmaB des Verfalls kritischen Bewußtseins, wenn Theodor $\$$. Adorno, Ludwig v. Friedeburg, Heinrich Böll, Harry Pross - vom . Willen (erfüllt), jenen Irrwegen zu begegnen, die einst eine unselige Zeit (!) möglich madten - sich in der Gesinnung *elementarer Menschlichkeit, Oberzeugungstreue und rechtsstaatlichen Denkensa und im >Glauben (!) an unsere demokratische Staatsordnung und ein nach innen und außen befriedetes Europa (!) a mit Hermann J. Abs, Ernst Benda, Julius Kardinal Döpfner, Freiberr zu Gurtenberg, Ernst Lemmer und Herbert Wehner vereinen.

6 Zur Entstehungsgeschichte des Grundgesetzes: Jahrbuch des öffenclichen Reches der Gegenwart, Neue Folge Bd. I, hrsg. von Gerhard Leibholz und Hermann v. Mangoldt, Entstehungsgeschichte der Arrikel des Grundgesetzes, bearbeiter von $\nabla$. Doemming, Füsslein, Matz. - Zum Hintergrund der politischen Auseinandersetzungen bei der Ausarbeitung der Verfassungsordnung vgl. neuestens die eingehende Untersuchung von Werner Sörgel, Konsensus und Interessen, Eine Studie zur Entstehung des Grundgesetzes für die Bundesrepublik Deutschland, Stuttgart 1969. - Sörgel weist zutreffend darauf hin (S. I 5), daß nach dem Zerfall der Kriegskoalition mit der UdSSR das Interesse der westlichen Besatzungsmächte primär darauf gerichtet war, in Frontstellung zum Ostblods die politischen und wirtschaftlichen Verhältnisse Westeuropas zu konsolidieren. Vor allem den USA erschien dazu die Errichrung eines westdeutschen Teilstaates notwendig. Sie glaubten, auf das wirtschaftliche Potential Westdeutschlands für ihre Strategie des rolling badk niche verzichten zu können.

$\checkmark$ Vgl. den Text der Frankfurter Dokumentee, abgedr. im Bonner Kommentar, Hamburg r9so ff. Einleitung S. $39 \mathrm{ff}$.

a Vgl. Sörgel (Anm. 6), S. r rof.

- Nur CDU, CSU, FDP und SPD haben sich an allen fünf Bundestagswahlen beteilige. Im r. Bundestag waren ro Parteien, I Wahlblodsvertreter und 2 Unabhängige vertreten. Insgesame haben sich 1949, 1953, 1957, 1961 und 1965 31 verschiedene Parteien zur Wahl gestellt. Die in den Führungsspitzen von CDU/CSU und SPD diskutierte Wahlrechesreform soll die Monopolstellung der beiden Parteien verfassungsrechtlich verewigen. Ob ein verfassungsänderndes $\mathrm{Ge-}$ setz mit dem Ziel, das Mehrheitswahlrecht einzuführen, vor dem Bundesverfassungsgericht Bestand hat, ist eine andere Frage. 
Der Theorie nach sollten die Parteien allerdings einerseits Instrument der Vermittlung gesellschaftlicher Interessen und Bedürfnisse und andererseits das Medium rationaler innerparteilicher Auseinandersetzung sein, um in Diskussionen den aufgeklärten Willen der Mitglieder artikulieren und erzeugen zu helfen ${ }^{10}$. Fakrisch wurde jedoch das Verfassungsgebot, daß ihre innere Organisation demokratischen Grundsätzen entsprechen muß (Art. 2 I Abs. I Satz 3 GG), um den Wahlbewerbern für den Bundestag eine ausreichende demokratische Legitimation zu vermittein, in der Folgezeit wirksam unterlaufen. Immerhin war dem Ansatz nach in dieser Verfassungsbestimmung das Gebot der Demokratisierung gesellschaftlicher Organisationen enthalten.

Für den historischen Kompromißcharakter des Grundgesetzes kennzeichnend ist, daß über die zentrale Frage, weiche politischen und organisatorischen Konsequenzen aus der Proklamierung des Sozialstaatsgebots sich ergeben müßten, trotz der unmittelbar präsenten Erfahrung des Scheiterns der Weimarer Republik Einigkeit nicht zu erzielen gewesen war. Das Pathos des Ahlener Programms von 1947 , daß $»$ das kapitalistische Wirtschaftssystem den staatlichen und sozialen Lebeninteressen des deutschen Volkes nicht gerecht geworden ist«, hatte die Christiche Einheitspartei 1949 bereits verdrängt. SPD und KPD hatten die Uberwindung der bloß formal verstandenen parlamentarischen Demokratie, bei der sich Demokratie in die technische Verfahrensweise einer Rationalisierung des politischen Prozesses ${ }^{11}$ verflüchtigt, als Forderung $z$ war verbal noch angemelder, zu konkreten Aktionen, z. B. Streiks zur Durchsetzung der verfassungsmäßigen Anerkennung demokratischer Teilhaberechte in allen gesellschaftichen Bereichen kam es jedoch nicht ${ }^{12}$. Dahinter stand zumindestens bei der SPD und den Gewerkschaften die optimistische Erwartung, daß auf dem Wege über die Parlamentsmehrheit die durch dilatorische Formelkompromisse offengelassenen Machtfragen parlamentarisch entschieden werden könnten. Hinzu kommt, daß nach dem Zusammenbruch im Zeichen einer allgemeinen Mangelwirtschaft ein scheinbarer Abbau von Herrschaftsverhältnissen vor sich gegangen war. Demontagedrohungen hatten in dieser Phase zu einer Einheitsfront der sozialen Gegenspieler geführt.

Dieser Entwidklung entsprach die zügige Eingliederung nahezu der gesamten früheren Beamtenschaft des NS-Staats in den Staatsapparat der Bundesrepublik mit allen ihren überkommenen rechtlich geschützten Positionen ${ }^{13}$.

Es erstaunt rüdkblickend nicht, daß bei dieser Ausgangssituation im Zuge der unmittelbar mit der Gründung der Bundesrepublik einsetzenden Restaurationsperiode die verfassungsrechtliche Möglichkeit einer sozialen Umgestaltung der gesellschaftlichen Verhältnisse ungenutzt geblieben ist. Der Sozialisierungsartikel des Grundgesetzes (Art. I 5 ), wonach Grund und Boden, Naturschätze und Produktionsmittel zum $Z$ weck der Vergesellschaftung in Gemeineigentum oder in andere Formen der Gemeinwirtschaft überführt werden können, blieb ebenso wie der Verfassungssatz, daß Eigentum verpflichtet und sein Gebrauch zugleich dem Wohl der Allgemeinheit zu dienen hat (Art. I4 Abs. 2 GG), ohne praktische Folgen. Spätestens bei der Verabschiedung des Betriebsverfassungsgesetzes im

$10 \mathrm{C}$. Offe, Politische Herrschaft und Klassenstrukruren. Zur Analyse spärkapiralistischer Gesellschaftssysteme. In: Kress u. Senghaas, Hrsg., Politikwissenschaft, Eine Einführung in ihre Probleme, Frankfurt/Main 1969, S. 155-189 (S. 166 f.).

11 Vgl. K. Hesse, Grundzüge des Verfassungsrechts der Bundesrepublik Deutschland, 3. Aufl., Karlsruhe 1969 , S. 55 .

12 Sörgel zufolge haben die Gewerkschatten das Zustandekommen des Grundgesetzes 1949 nahezu uneingeschränkt begrüßr, a. a. O. (vgl. Anm. 6), S. II.

13 Verfassungsrechelich war der Weg dazu durch die Formulierung des Art. 13 I GG geebner. 
Jahre I952 wurde die politische Ohnmacht der Interessenvertretungen der Arbeiterschaft offen dokumentiert. Es entbehrt nicht einer gewissen Ironie, wenn der CDU-Generalsekretär Heck die objektive Funktion der SPD in den ersten Jahren der Bundesrepublik rückblickend so charakterisiert: $\gg D i e$ SPD hatte die Aufgabe, die oppositionellen $\mathrm{Kräfte}$ in unserem Land, besonders aber die linken Exureme, zu binden und sie so an der Zerstörung der jungen Demokratie zu hindern. Daß den Sozialdemokraten dies gelungen ist, gehört zu ihren ganz großen Leistungen. «14 Heute läßt sich sagen, daß mit der Tabuierung des ökonomischen status quo eine der entscheidenden Bedingungen für die spätere politische Entwidklung der Bundesrepublik gesetzt worden ist.

\section{III.}

Die liberale Hoffnung, die verfassungsrechtliche Anerkennung und Sicherung formaldemokratischer Verfahrensregelungen und rechtsstaatlicher Grundsätze, vor allem die grundrechtlichen Freiheitsverbürgungen würden zu einem Ausbau demokratischer Ansätze führen, diese liberale Hoffnung trog.

Die Chancen, die das Grundgesetz mit seinen Formelkompromissen noch bot, wurden nicht genutzt. Der These Helmut Ridders ist zuzustimmen, daß die Verfassung bereits im Augenblick ihres Eintritts in den politischen Prozeß "links" von der gesellschaftlichen Wirklichkeit gestanden hat, weil die Verfassungsschöpfung nicht revolutionär mit einer tiefgreifenden Umwandlung vordemokratischer gesellschaftlicher Verhältnisse verbunden gewesen ist ${ }^{15}$.

Die weitgehend geschlossene Konzeption des liberalen Verfassungsmodells mit der nicht unwichtigen sozialstaatichen Komponente wurde in einem I95I beginnenden Prozeß aufgebrochen. Verfassungspolitisch lassen sich mehrere Phasen im bundesrepublikanischen Restaurationsprozeß erkennen.

r. Das politische Programm der herrschenden Kräfte ist in der Entstehungsphase der Bundesrepublik auf die Konsolidierung der politischen und wirtschaftlichen Verhältnisse gerichtet; es zielt im Kern auf die Verteidigung der trotz des $\mathrm{Zu}$ sammenbruchs von I 945 nach wie vor bestehenden kapitalistischen Gesellschaftsordnung hin. Nach außen gilt es, die als real begriffene kommunistische Bedrohung abzuwehren; nach innen, der damals noch als »revolutionär« mißverstandenen Sozialdemokratischen Partei keine Chance für einen Wechsel in der Führungsspitze zu geben.

Außenpolitisch findet diese Situation in der vorbehaltlosen Unterordnung unter die Interessen der amerikanischen Politik ihren Ausdruck. Die Erwartungen, die insbesondere die USA an die Gründung des kapitalistischen Frontstaates geknüpft hatten, wurden damit voll erfüllt. Innenpolitisch erweist sich die KoreaKrise als Alibi für die im Jahre r95 I beschlossenen Bestimmungen des politischen Strafrechts, die großenteils verfassungswidrig sind und sich unmittelbar auf nationalsozialistische Vorbilder zurüdkführen lassen ${ }^{16}$. Mit formal-demokrati-

13 Zitiert im Spiegel Nr. 26 vom 23. 6. 1969 S. 31 . Zur Rolle der SPD in der politischen Entwidslung Westdeutschlands vgl. J.Schaltenbrand, Die SPD in der gesellschaftlichen Entwidklung nach dem 2. Weltkrieg. In: neue kritik I962, Nr. 10 S. 7-I4.

$15 \mathrm{H}$. Ridder, Thesen zum Thema •Verfassung und Verfassungswirklichkeit*, vorgelegt dem Giessener Arbeitskreis > Kritische Recheswissenschatt«, SS 1969, hektographiert.

16 Durch das auf dem Höhepunke des Kalten Kriegs beschlossene Strafrechtsänderungsgesetz vom 30. 8. I95 I wurde die strafrechtiche Verfassungsschutzbestimmung des Art. 143 GG außer Kraft gesetze. Zum politischen Strafreche vgl. Copič, Grundgesetz und politisches Strafrecht never Art, Tübingen 1967. Die Novellierung des politischen Strafrechts durch das 8. Straf- 
schen Mitteln war damit für die staatlichen Instanzen eine Handhabe geschaffen, den politischen Gegner, der die Gesellschaftsordnung zu verändern versucht, wirksam bekämpfen zu können. Die Kriminalisierung von Minderheiten hat sich allemal als wirksames Mittel in der innenpolitischen Auseinandersetzung erwiesen. Das politische Klima jener Zeit führte zu einer Fülle von politischen Strafprozessen, die objektiv Momente des psychischen und physischen Terrors enthielten. Sie richteten sich in erster Linie gegen Betriebsräte und Mitglieder der Kommunistischen Partei, denen zum Teil nur Kontakte mit staatlichen Instanzen und gesellschaftlichen Organisationen der DDR zum Vorwurf gemacht werden konnten. Schon vor dem Verbot der KPD wurde der westdeutsche Staatsapparat von Anhängern dieser Partei und ihr nahestehender Organisationen gesäubert. Auch beim DGB hielt man die Zugehörigkeit zur KPD mit der Mitgliedschaft in Gewerkschaften für unvereinbar. In logischer Konsequenz ließ das politische Selbstverständnis im Jahre 1956 das legale Verbor jener politischen Partei zu, zu deren Programm die Veränderung der politischen und gesellschaftlichen Wirklichkeit gehörte. Mit dem KPD-Verbotsurteil17 wurde das verfassungsrechtlich legitimierte Freund-Feind-Denken Carl Schmitts vollzogen. Man sollte sich vergegenwärtigen, daß spätestens seit jener Zeit die Ausschaltung, ja sogar die Vernichtung des innenpolitischen Gegners Bestandteil der politischen Praxis der Bundesrepublik geworden ist.

Wenn demgegenüber auf das Verbot der Sozialistischen Reichspartei im Jahre $1952^{18}$ hingewiesen wird, so sollte über die tatsächliche Funktion dieser Verbotsmaßnahme kein Zweifel bestehen. Das Verbot der Sozialistischen Reichspartei ist Bestandteil eines von oben gesteuerten kollektiven Verdrängungsprozesses. Symprome dieses Verdrängungsprozesses sind die Marginalisierung der NSGewaltverbrechen und deren Interpretation als volksfremde Geschehnisse sowie, damit korrespondierend, die These von der Verführung des im Kern anständigen Volkes durch seine falschen Führer. Die Bestrafung nationalsozialistischer Straftäter entspricht in vielen Fällen der für Betrüger im Rüdkfall; fast scheint es, als ob die bürgerliche Gesellschaft Eigentumsdelikte als Verstoß gegen ihr materielles Prinzip unnachsichtiger ahndet als die Verbrechen des Faschismus. Kennzeichnend für die lediglich formaljuristische geleistete Aufarbeitung der nationalsozialistischen Vergangenheit ist, daß man die wahren Ursachen der NS-Herrschaft nicht aufzuklären sucht. $\mathrm{DaB}$ im starren Bezugssystem justizförmigen Denkens ein inhaltlicher Begriff von Demokratie als Gegenposition zum Faschismus sich nicht entwickeln läßt, verwundert deshalb nicht. Die offizielle Rechtfertigungsideologie bewältigt die NS-Vergangenheit mit Phrasen wie »die Ereignisse der Geschichte (werden) aus der Tiefe des Irrationalen gespeist « (Kiesinger $)^{19}$ und lenkt so von den gesellschaftlichen Verbältnissen, die den Nazismus hervorgebracht hatten, systematisch ab.

2. Den zweiten Einschnitt in der verfassungsrechtlichen Entwidklung stellt das verfassungsändernde Gesetz zur Wiederbewaffnung der Bundesrepublik im Jahre I956 dar. Die klare, gegen jede Remilitarisierung des westdeutschen Teil-

rechtsänderungsgesetz vom 25.6. 1968 hat an den verfassungsrechtlichen Bedenken nichts geändert.

17 BVerfGE s, 85 ff. DaB das KPD-Verbotsurteil eine politisch bedeutungslos gewordene Partei ausschaltere, steht auf einem anderen Blatt.

$18 \mathrm{BVerfGE} 2$, I ff.

19 Bundeskanzler Kiesinger in seiner vor dem Kongreß der Handelsvertreter und Handelsmakler am 3.6. 1969 in Bonn gehaitenen Ansprache Bewahrung des inneren und äußeren Friedens*, Bullerin des Presse- und Informationsamtes der Bundesregierung Nr. 72 v. 7.6. 1969 S. $621-624$ (S. 624). 
staates getroffene Verfassungsentscheidung wurde damit eindeutig verletzt. In

den Beratungen des Parlamentarischen Rates hatte man noch für selbstverständlich gehalten, daß die Bundesrepublik keine Wehrmacht haben soll und die Sicherheit der Bundesrepublik in anderer Weise als durch das nationale militärischindustrielle Machtpotential zu garantieren ist 20.

In gewisser Weise hat die Wiederbewaffnung eine Schlüsselfunktion, weil damir erstmals unverhüllt ein entscheidender Bezugspunkt in der Verfassungskonzeption des Grundgesetzes von 1949 eliminiert worden ist. Hinzu kommt: eirraus Gründen der militärischen Sicherheit ausufernder Geheimbereich schließt die öffentliche Diskussion über zentrale politische Fragen aus; es wird zusätzlich ein Bereich geschaffen, in dem die Parlamentskontrolle zur sinnentleerten Akklamation der Arbeit weitgehend verselbständigter Ministerialbürokratien regrediert. Gegenüber der Trias von Berufspolitikern, Staatsbürokratie und Militär läßt sich ein wirksames System der Gewaltenkontrolle nicht installieren. Die beherrschende Rolle militärischen Denkens drüdkt sich in einer zunehmenden Militarisierung des öffentlichen Lebens aus ${ }^{21}$. Die Verstärkung des militärischen Einflusses kann darauf zurüdkgeführt werden, daß das Gewicht der Armee nicht nur von der latenten oder offenen Gewalt herrührt, die die militärische Matht verleiht, sondern auch von der wirtschaftichen Funktion der Armee bei der Vergabe von Aufträgen und ihrer Rolle bei der Weiterentwicklung der technischen Forschung ${ }^{22}$. Die I956 eingeleitete Remilitarisierung der Bundesrepublik ist der systematische Anfang des notwendigen Endes einer Illusion: der Illusion, daß es gelingen könne, Demokratie gesellschattsneutral als formales politisches Organisationsprinzip zu institutionalisieren.

3. Die Verabschiedung der Notstandsgesetze im vergangenen Jahr ist ein weiterer zentraler Punkt in der allgemeinen restaurativen Entwidklung. Bereits im Jahre I95s wurden im Zuge der Beratungen der Wehrverfassung die gesetzgeberischen Vorarbeiten für eine Notstandsverfassung von der Bundesregierung aufgenommen. Es schien ihr jedoch nicht opportun, diese in die Diskussion über die Remilitarisierung, deren notwendige Ergänzung sie ist, einzubeziehen ${ }^{23}$. Die Sozialdemokratische Partei hatte schon früh in einem Parteitagsbeschluß (Hannover I960) ihre Bereitschaft erklärt, win einer von staatspolitischer Verantwortung getragenen Haltung « frei von »parteiegoistischen Winkelzügen « an der zur »Lebensfrage $\propto$ stilisierten Notstandsverfassung konstrukriv mitzuarbei$\operatorname{ten}^{24}$.

Unter dem Mantel formal rechtsstaatlicher Stringenz und dem Vorwand, von der Verfassungswirklichkeit geforderte technisch notwendige Rationalisierungen vornehmen zu müssen, wurden die Kompetenzen der Exekutive einseitig zu Lasten der politischen und sozialen Rechte des Bürgers vergrößert und der Bedeutungsverlust des Parlaments als Kontrollinstanz gegenüber Regierung und

20 So Carlo Schmid in den Beratungen zu Art. 24 GG, vgl. จ. Mangoldt, Das Bonner Grundgesetz, I953, Art. 24 Anm. 4.

21 Dazu gehört, daß tendenziell alle öfentlichen Bereiche sich den Erfordernissen militärstrategischen Kalküls unterwerfen müssen. Sinnfälliger Ausdruck hierfür ist, daß im Haushalesplan des Bundes von 1969 der Verteidigungsetat mit 19 Milliarden DM den größten Haushaltsposten eingenommen hat. Daß damic Prioritätsentscheidungen gegenüber allen nid̆tmilitärischen Bereichen rerbunden sind, liegt auf der Hand.

22 Die Revolution und der Staat, Interview mit Henri Lefabvre, Naddrudx in sexpress internationala Nr. 77 vom 25. 7 . 1969.

93 Zur Entstehungsgeschichte der Notstandsgesetze $\nabla g 1$. D. Sterzel in: Kritik der Notstandsgesetze, Frankfurt/Main, 2968 S. 7 ff.

24 Beschluß des SPD-Parteitags in Hannover zur Notstandsgesetzgebung, abgedr. bei Friedrich Schäfer, Die Notstandsgeserze, I 966, S. 46 u. S. I I. 
staatlichen Bürokratien verfassungsrechtlich sanktioniert. Neben die vorbeugende Notstandsvorsorge, die verfassungsrechtlich bislang schon durch Parteienund Vereinigungsverbot (Art. 2 I und Art. 9 Grundgesetz) sowie durch die individuelle Grundrechtsverwirkung (Art. I 8 Grundgesetz) möglich gewesen ist, tritt nunmehr ein Instrumentarium zur gewaltsamen Unterdrüdkung oppositioneller Bewegungen und Kräfte. Die zusätzlich geschaffenen Möglichkeiten des Einsatzes der Bundeswehr im Innern und die Zivildienstverpflichtung der arbeitenden Bevölkerung kennzeichnen die konkrete Bedrohung, die - über das gängige Arsenal staatlicher Repressionsmittel hinaus - für den einzelnen vom Staat künftig in Konflikrsituationen ausgehen können. Auch wenn das Norstandsrecht nicht angewandt wird, dient es zur Einschüchterung oppositioneller Bewegungen und Intentionen. Rainer Barzel hat die politische Funktion der Notstandsgesetze aus der Sicht der CDU so beschrieben: sie sind »die wahre Regierungsbasis für das Deutschland von morgen «, »so gestärkt können wir dann jeder Belastung, die auf die Bundesrepublik zukommr, in nationaler Sammlung entgegensehen ${ }^{25}$.

Die Norstandsgesetzgebung hat das parlamentarische Regierungssystem in der Bundesrepublik von einer Seite beleuchtet, die sonst nicht ohne weiteres sichtbar ist: daß nämlich im Repräsentativsystem des Grundgesetzes die Souveränität des Volkes gebrochen ist. Die Notstandsverfassung ist eine besonders krasse Form des legalisierten Mißtrauens gegenüber dem Souverän; das Mißtrauen verwandelt sich hier in die legalisierte Form der Repression. Unverkennbar kommen die Notstandsgesetze damit zugleich der Hauptaufgabe des spätkapitalistischen Staates zugute, den status quo der gesellschaftlichen Herrschaftsstrukturen auch über Phasen ökonomischer Krisen und sozialer Konflikte hinweg mit systemkonformen, formaldemokratisch legitimierten Mitteln zu stabilisieren.

4. Die gegenwärtige Situation wird bestimmt durch den Versuch, die letzten Reste der föderalistischen Struktur der Bundesrepublik vollends zu beseitigen. Die Finanzreform und eng damit verbunden die angestrebte zentralisierte Wissenschaftsplanung laufen im Ergebnis darauf hinaus, den Ländern letzte originäre politische Kompetenzbereiche ("Finanzhoheit» und *Kulturhoheit«) zu nehmen.

Damit findet eine schon seit langem zu beobachtende Tendenz, den Ländern den Status von Vollzugsorganen des Bundes zuzuweisen, ihren Abschluß. Worauf diese Gleichschaltung der Länder zielt, liegr auf der Hand. Ungleichzeitigkeiten zwischen den einzelnen Ländern und im Verhältnis zum Bund, liberale Enklaven bedeuten Sand im Getriebe der sich verschärfenden allgemeinen autoritären Entwidklung.

IV.

Es bleibt zu fragen: Welche Konsequenzen sind im Rahmen einer verfassungsrechtlichen Diskussion aus der gegenwärtigen Verfassungslage zu ziehen?

Im Hinblick auf die aktuellen politischen Auseinandersetzungen kommt es darauf an, sich des materiellen Gehalts der Grundrechte zu erinnern. Die liberalen Freiheirsrechte haben im Zuge der Vergesellschaftung des Staates und der Verstaatlichung der Gesellschaft als bloße negative Abwehrrechte ihren historisch begründeten Sinn verloren. Begreift man diese Rechte aber als historische Rechte, die

25 Zitiert nach Schreiber, Die CDU geht aufs Ganze, in: Blätter für deutsche und internationale Politik, 1965, S. 507. 
einen möglichen Stand politischer Emanzipation verfassungskrättig normieren, so läßr sich ihr Inhalr nur im Zusammenhang des Gesamtzustands der Gesellschaft hinreichend bestimmen ${ }^{20}$.

Sollen unter den Bedingungen einer technokratisch verwalteten Klassengesellschaft Grundrechte den durch sie garantierten Freiheitsbereich tatsächlich gewährleisten, so lassen sie sich nur als soziale Teilhaberechte begreifen.

Die studentische Protestbewegung ist die Reaktion auf den gescheiterten Versuch innerhalb der politischen Institutionen, der Bundesrepublik systemimmanent Reformziele zu formulieren und öffentlich zur Anerkennung zu bringen. Die demokratische Opposition versuche, durch Symbolangriffe die neuralgischen Punkte der Gesellschaft sichtbar und dadurch Offentlichkeit erst wieder möglich zu machen. Die Aktionen der Außerparlamentarischen Oppositionen erhalten ihren politischen Sinn aus der Intention, den Widerspruch von scheinbarer Integrität der Verhältnisse und dem inhaltlichen Anspruch von Demokratie - herrschaftsfreie, durch die objektiven Interessen der Menschen voll legitimierte Ordnung zu sein - zu entlarven. Die Forderung, die autoritär deformierte Demokratie durch Selbstbestimmungsrechte in allen gesellschaftlichen Teilbereichen zu demokratisieren, nimmt das sozialstaatliche Versprechen des Grundgesetzes beim Wort und geht von der Einsicht aus, daß anders Menschenwürde sich nicht verwirklichen läßt' ${ }^{27}$.

26 O. Negt hat den Funktionswandel der. Grundrechre an Hand ron Art. s Abs. I und Arr. 8 GG im Rahmen einer gutachtlichen Außerung im Prozeß gegen Thomas Schmitz-Bender vor dem Landgericht in München (Demonstration vor dem griechischen Konsulat in München) exemplifriert; eine überarbeitete Fassung des Gutachtens ist abgedrucke in: Die neue Linke, r969, Heft $x$.

27 Vgl. R. Smend, der in seinern Beitrag $\gg$ Deutsche Staatsrechtswissenschaft vor hundert Jahren - und heute darauf hinweist, daB die Verfassungstheorie > Erscheinungen wie die der * Außerparlamentarischen Opposition« oder der Mitbestimmung - im Betrieb, im Parteiengesetz - (nur dann) sachgemäß zu erfassen vermag, wenn sie die Forderung, >daß der Staat rom Menschen und seiner Bestimmung her verstanden werden mußa, zum Ausgangspunkt ihrer Oberlegungen nimmt. In: Festschrift für Adolf Arndr, Frankfurt/M., 1969 S. 45I-461 (S. 460/x). 